\title{
ESTUDO COMPARATIVO ENTRE OS CONTROLADORES FUZZY E PID APLICADOS AO CONTROLE DE UM PÊNDULO INVERTIDO UTILIZANDO PLATAFORMA LEGO MINDSTORMS NXT
}

\author{
Hugo V. D. Silva*, Wylliam S. Gongora*, Rodrigo R. Sumar*, Marcos B. R. Vallim*, \\ Alessandro Goedtel* \\ * Universidade Tecnológica Federal do Paraná - UTFPR \\ Programa de Pós-Graduação em Engenharia Elétrica \\ Av. Alberto Carazzai, 1640, Cornélio Procópio, Paraná, Brasil
}

Emails: a1218735@pos.utfpr.edu.br, a1035258@pos.utfpr.edu.br, sumar@utfpr.edu.br, mvallim@utfpr.edu.br, agoedtel@utfpr.edu.br

\begin{abstract}
The inverted pendulum on car is a system used in the study of theories of control. Their nonlinear dynamics allows both the application of controls classical and intelligent system. This article has the objective to contribute to the study of nonlinear systems, presenting a comparative of conventional and fuzzy controller applied to stabilize the inverted pendulum in the car. This contribution is given by the development control rules otimizidas, aimed at reducing execution time and implementation in a device with low processing power. In this work, the inverted pendulum was built using the Lego Mindstorms $\mathrm{NXT}^{\circledR}$ kit and modeled, and performance of the controllers is analyzed. Experimental results are presented to validate the proposal.
\end{abstract}

Keywords— Fuzzy logic, inverted pendulum, Lego Mindstorms NXT ${ }^{\circledR}$.

Resumo- O pêndulo invertido sobre carro é um sistema utilizado no estudo das teorias de controle. Sua dinâmica não-linear permite tanto a aplicação de controles clássicos quanto de sistema inteligentes. Este artigo visa contruibuir para os estudos de sistemas não-lineares, apresentando um comparativo do controlador convencional e o nebuloso, aplicados à estabilização da haste do pêndulo invertido sobre carro. Tal contribuição é dada pelo desenvolvimento de regras de controle otimizidas, visando a redução do tempo de execução e a implementação em um equipamento com baixo poder de processamento. Neste trabalho, o pêndulo invertido foi contruído utilizando o kit Lego Mindstorms NXT ${ }^{\circledR}$ e modelado, e o desempenho dos controladores é analisado. Resultados experimentais são apresentados para validar a proposta.

Palavras-chave- Lógica fuzzy, pêndulo invertido, Lego Mindstorms NXT ${ }^{\circledR}$.

\section{Introdução}

O estudo da dinâmica de movimentos é útil em diversos ramos de pesquisa, tendo como uma de suas vertentes a análise dos sistemas oscilatórios. Com o objetivo de explorar a dinâmica de tais sistema, o pêndulo simples é utilizado, pois desenvolve seus movimentos em torno de uma posição de equilíbrio.

A variação deste sistema é conhecido como pêndulo invertido, que possui a sua massa acima do seu ponto fixo. Este sistema está relacionado, por exemplo, ao controle de foguetes, mísseis, plataformas e naves aeroespaciais em que o centro de gravidade está localizado por trás do centro de arrasto aerodinâmico, o que resulta na instabilidade (Dorf e Bishop, 1998).

Devido as suas características não-lineares e por ser um problema multi-variável conforme Fun e Lin (2005), admite-se que o pêndulo invertido é instável, pois seu sistema deve ser continuamente equilibrado, a fim de permanecer na posição vertical.

O pêndulo invertido é um sistema clássico de controle, onde as primeiras pesquisas na área são apresentadas por Furuta (1976). Tais sistemas são utilizados para testar a dinâmica e a teoria de controle (controladores clássico, redes neurais, controle fuzzy, algoritmos genéticos, etc.).
Montado algumas vezes sobre carros, este modelo possui uma base móvel onde os motores são responsáveis pelos movimentos horizontais que mantém a haste do pêndulo estabilizada (Morais et al., 2005; Wu et al., 2008; Faizan et al., 2010).

Por se tratar de um sistema que possui regiões de instabilidade, uma solução que atenda toda a sua dinâmica, nem sempre é possivel. No entanto, considerando linear apenas uma região próxima ao ponto de equilíbrio do pêndulo, um controlador Proporcional-Integral-Derivativo (PID) pode ser aplicado para a estabilização, como visto em Faizan et al. (2010).

Outros trabalhos realizam uma comparação entre os métodos de controle, quando aplicados a estabilização do pêndulo invertido construído sobre um carro (Nour et al., 2007; Yadav et al., 2011).

Este artigo tem como objetivo contribuir para os estudos de sistemas não-lineares, comparando o desempenho entre o controlador PID e o controlador fuzzy quando aplicados ao sistema do pêndulo invertido sobre carro. Tal sistema é construído sobre a plataforma Lego Mindstorms $\mathrm{NXT}^{\circledR}$, que dispõe de um bloco programável onde as rotinas de controle são embarcadas.

Este trabalho está dividido da seguinte maneira: na Seção 2 são apresentados as características do sistema bem como sua modelagem mate- 
mática. Na Seção 3 é detalhado o protótipo com os sensores e atuadores utilizados. Na Seção 4 as característica dos controladores, o equacionamento e as regras de implicação são descritas. Já na Seção 5 uma abordagem da metodologia utilizada é relatada. Finalizando, a Seção 6 apresenta os resultados experimentais e a conclusão.

\section{Pêndulo invertido}

Os pêndulos invertidos sobre carros são construídos das mais diversas formas. Sendo implementado em vários equipamentos de controle, tal experimento é útil no estudo das técnicas de estabilização e modelagem de sistema dinâmicos (Nise, 2002; Ogata, 2010).

Para as aplicações e estudos neste trabalho é necessário o desenvolvimento de uma modelo matemático que represente a dinâmica do sistema real.

\subsection{Modelagem matemática}

O modelo físico o qual toma como referência o pêndulo invertido sobre carro descrito em Ogata (2010), é apresentado na Figura 1.

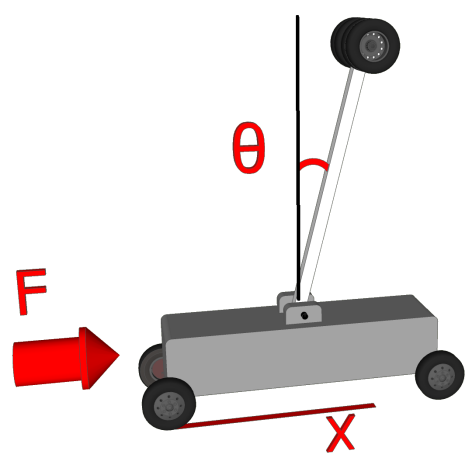

Figura 1: Sistema do pêndulo invertido

Neste sistema, o movimento rotacional da haste do pêndulo em relação ao centro de gravidade da mesma é dada pela equação (1) e o movimento horizontal do carro é descrito pela equação (2).

$$
\begin{gathered}
I \ddot{\theta}=V l \sin \theta-H l \cos \theta \\
M \ddot{x}=u-H
\end{gathered}
$$

Tem-se para a equação 1 que $I$ é o momento de inércia da haste em relação ao centro de gravidade, $V$ e $H$ são o movimento vertical e horizontal da haste respectivamente, em relação centro de gravidade, $l$ é a distância do carro ao centro de massa do pêndulo e o $\theta$ é o ângulo gerado entre a base do carro e a haste do pêndulo. Já para a equação 2 tem-se que $M$ é a massa do carro, $u$ a força aplicado sobre a base do carro e $x$ o deslocamento do carro em relação a sua posição inicial.
O objetivo do controle é manter o pêndulo invertido na posição vertical, impondo à haste do pêndulo a miníma variação possível. Logo, admite-se que $\theta(t)$ e $\dot{\theta}(t)$ são valores pequenos e aproxima-se $\sin \theta \cong \theta, \cos \theta \cong 1$ e $\theta \cdot \dot{\theta}^{2}=0$ para pequenas variações angulares. Sendo assim, podese reescrever as equações (1) e (2) da seguinte forma:

$$
\begin{gathered}
(M+m) \ddot{x}+m l \ddot{\theta}=u \\
m l^{2} \ddot{\theta}+m l \ddot{x}=m g l \theta
\end{gathered}
$$

Utilizando as equações (3) e (4), obtém-se a função de transferência descrita na equação (5):

$$
\frac{\Theta(s)}{-U(s)}=\frac{1}{M l\left(s+\sqrt{\frac{M+m}{M l} g}\right)\left(s-\sqrt{\frac{M+m}{M l} g}\right)}
$$

Os parâmetros físicos do sistema, contidos na Tabela 2, são utilizados para descrever o comportamento dinâmico do pêndulo invertido construído sobre carro.

\begin{tabular}{clc}
\multicolumn{2}{c}{ Tabela 1: Parâmetros Físicos do Sistema } \\
\hline \hline \multicolumn{2}{c}{ Descriçấo } & Valor \\
\hline$g$ & Aceleração da gravidade & $9,81 \mathrm{~m} / \mathrm{s}^{2}$ \\
$M$ & Massa do carro & $0,8 \mathrm{~kg}$ \\
$m$ & Massa do pêndulo & $0,2 \mathrm{~kg}$ \\
$l$ & Comprimento da haste & $0,4 \mathrm{~m}$ \\
$d$ & Diâmetro das rodas & $0,04 \mathrm{~m}$ \\
\hline \hline
\end{tabular}

Um diagrama esquemático que representa o modelo físico é apresentado na Figura (2). Neste modelamento tem-se que a tensão $(u(t))$ aplicada a função de transferência do Motor de Corrente Contínua (MCC) é convertida em torque mecânico $(T)$. Considerando que este torque é diretamento aplicado às rodas do carro, divide-se o valor de saída pelo diâmetro $(d)$ das rodas, obtendo-se a força $(F)$.

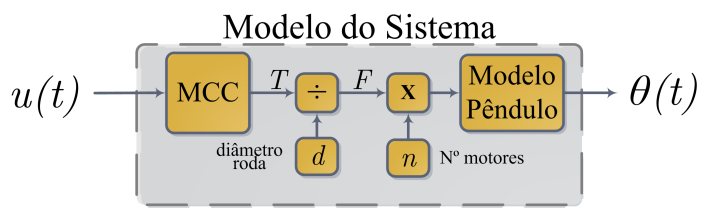

Figura 2: Diagrama de blocos do sistema

A força é composta pela atuação de dois motores e este sinal é multiplicado pelo número de atuadores $(n)$ utilizados. Finalizando a dinâmica do sistema, este sinal é apresentado ao bloco do modelo do pêndulo, que tem por resposta o ângulo $(\theta(t))$ da haste. 


\section{Protótipo do pêndulo invertido}

O pêndulo invertido sobre carro foi construído com o kit Lego Mindstorms NXT ${ }^{\circledR}$ utilizando as peça de plástico disponíveis no kit, o que proporciona uma rápida montagem do experimento e facilidade para realizar pequenos ajustes na estrutura.

O kit possui um bloco programável (brick) que dispõe de quatro entradas para sensores e três saídas para motores. Um processador $A R M 7^{\mathrm{TM}}$ é responsável pelo processamento, execução do algoritmo, gerenciamento dos drivers de controle dos motores e comunicação com os sensores externos.

$\mathrm{O}$ pêndulo invertido é posicionado sobre o carro por um eixo móvel, o qual repousa sobre mancais fixos no carro. Tal fixação limita o movimento da haste e a queda em um sentido.

$\mathrm{O}$ veículo possui dois motores na sua base, os quais realizam o movimento unidirecional com o objetivo de manter o pêndulo equilibrado. $\mathrm{Na}$ Figura 3 é apresentado o protótipo do sistema.

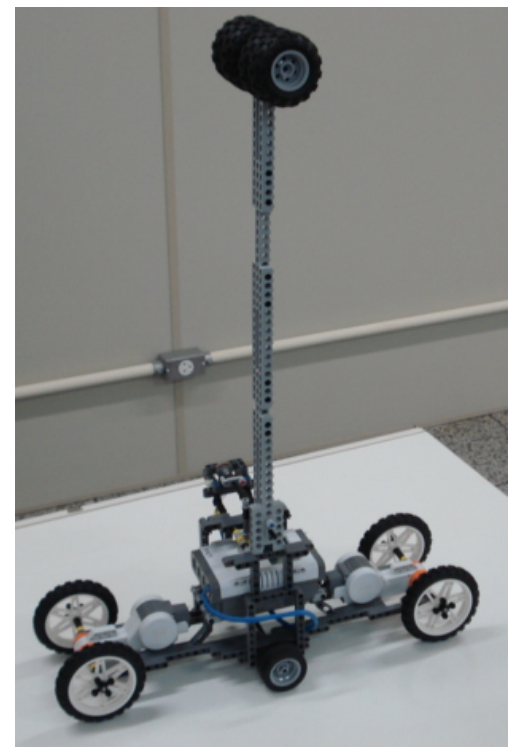

Figura 3: Pêndulo invertido sobre carro

\subsection{Sensor}

O kit Lego Mindstorms NXT ${ }^{\circledR}$ possui vários tipos de sensores, tais como sensor de toque, som, luminosidade, distância, entre outros. Mas para a construção deste trabalho foi desenvolvido um sensor de ângulo, que mede a posição do pêndulo em relação a base do carro.

Para este fim, um potenciômetro não-linear de $10 \mathrm{k} \Omega$ é ligado as entradas do conversor Analógico/Digital (A/D), conforme a Figura 4.

O conversor A/D do bloco programável realiza uma leitura da faixa de tensão aplicada aos pinos 1 e 2, produzindo um valor entre 0 e 1023 em escala binária.

O potenciômetro possui uma faixa de operação de 270 graus, porém a variação do ângulo da

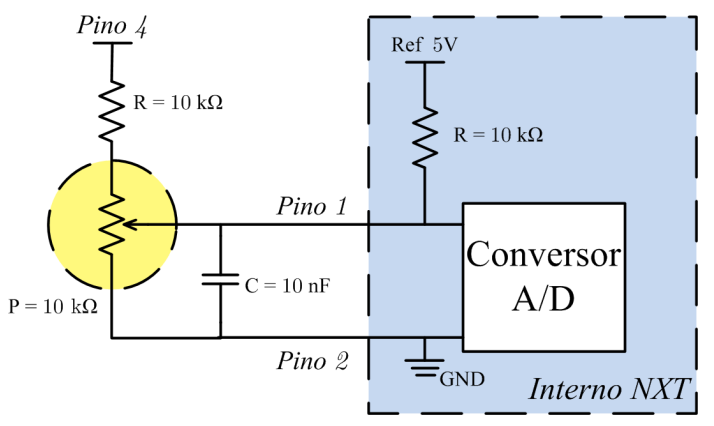

Figura 4: Esquema elétrico ligação potenciômetro

haste é fixada em 180 graus. Aplicando a haste do pêndulo uma variação de $-\pi / 2$ a $\pi / 2$, obtem-se a curva de resposta mostrada na Figura 5.

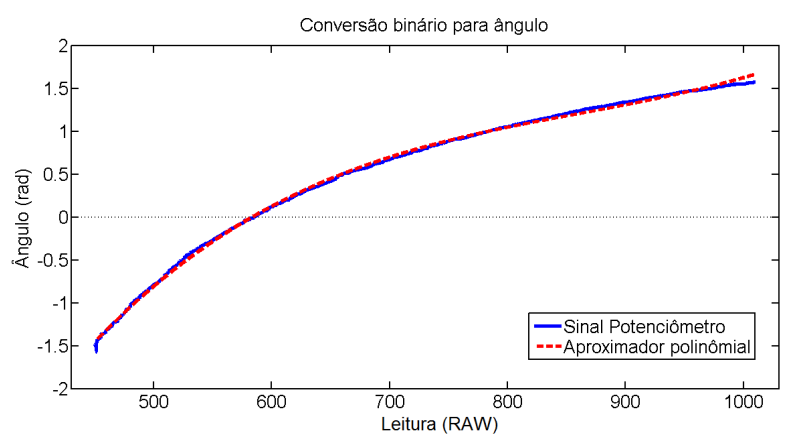

Figura 5: Resposta do sensor de ângulo

A linha pontilhada da Figura 5 é a referência para leitura do ângulo, a qual é o ponto de equilíbrio da haste. Logo, a variação da leitura é dada entre $-90^{\circ}$ e $+90^{\circ}$. Devido a característica não linear do potenciômetro, é realizado uma aproximação polinômio no sinal, obtendo a equação (6).

$$
y=2,32.10^{-8} x^{3}-5,99 \cdot 10^{-5} x^{2}+0,05 x-15,81
$$

Desta forma, a equação relaciona os valores lidos $(x)$ e o ângulo da haste $(y)$ em rad.

\subsection{Atuadores}

Neste trabalho foram utilizados dois MCCs disponíveis no kit, sendo que a tensão aplicada aos motores variam de $-7,4 \mathrm{~V}$ a $+7,4 \mathrm{~V}$, fornecida pela bateria de Níquel-hidreto metálico (NiMH).

O MCC pode assumir uma velocidade de até $170 \mathrm{rpm}$ quando aplicado a tensão máxima disponível na bateria (Gonçalves et al., 2010).

Considerando o equacionamento proposto por Krishnan (2001), pode-se calcular o torque $\left(T_{e}\right)$ do MCC relacionando a tensão $(e)$ e a corrente de armadura $\left(i_{a}\right)$, como a velocidade angular do motor $\left(\omega_{m}\right)$, conforme a equação $(7)$.

$$
T_{e}=\frac{e i_{a}}{\omega_{m}}
$$


Considerando por simplificação que o quociente da tensão pela velocidade angular trata-se de uma constante, $\left(K_{b}=e / \omega_{m}\right)$, pode-se transcrever a equação (7) para a equação (8).

$$
T_{e}=K_{b} i_{a}
$$

A dinâmica mecânica do MCC é apresentada na equação (9), onde o produto da derivada da velocidade angular $\left(d \omega_{m} / d t\right)$ pelo momento de inércia $(J)$ somado ao produto do coeficiente de atrito $(B)$ pela velocidade angular e igual ao torque de aceleração $\left(T_{a}\right)$, sendo que este é a diferença entre o torque eletromagnético $\left(T_{e}\right)$ é o torque de carga $\left(T_{l}\right)$.

$$
J \frac{d \omega_{m}}{d t}+B \omega_{m}=T_{a}=T_{e}-T_{l}
$$

Utilizando os parâmetros do MCC da Tabela 2, conforme Gonçalves et al.(2010), e aplicandoos na modelagem matemática do motor, pode-se calcular o torque gerado pelo MCC em funcionamento.

Tabela 2: Parâmetros físicos do MCC

\begin{tabular}{clc}
\hline \hline & \multicolumn{1}{c}{ Descrição } & Valores \\
\hline$K_{b}$ & Constante $(V / \omega)$ & 0,49 \\
$T_{c}$ & Torque de Carga & $3,55.10^{-4}$ \\
$B$ & Coeficiente de atrito & $1,92.10^{-3}$ \\
$R_{a}$ & Resistência de armadura & 7,6 \\
$L_{a}$ & Indutância de armadura & $4,88.10^{-3}$ \\
\hline \hline
\end{tabular}

\subsection{Linguagem de programação utilizada}

Para que a implementação das regras de controle seja possível, foi utilizada a linguagem leJOS que é baseada em Java ${ }^{T M}$. A linguagem leJOS é orientada a objeto, o que facilita a utilização de rotinas previamente construidas.

Para a criação e edição dos programas, utilizase a plataforma Eclipse IDE que também compila o programa gerado e o grava no bloco programável.

\section{Aspectos sobre controladores}

Para estabilização da haste do pêndulo são comparados dois métodos de controle, os quais buscam manter o ângulo da haste o mais próximo de sua referência, reduzindo assim o erro.

Sendo assim, é utilizado neste trabalho uma estratégia de controle atuando sobre o ângulo de variação da haste, desprezando o deslocamento do carro em relação a sua posição inicial.

\subsection{Controlador PID}

Os controladores PID são os mais utilizados nos sistemas de controle industrial, pois dispõe de uma gama de aplicações consideráveis além de uma fácil implementação física (Ogata, 2010).

Entretanto, sua aplicação necessita de um prévio conhecimento e modelagem do sistema em questão. $\mathrm{O}$ que possibilita o ajuste dos ganhos do controlador de modo off-line, como é o caso deste trabalho.

Os controladores PID são representados matemátimacamente pela equação (10) (Nise, 2002).

$$
G_{c}(s)=K_{p}\left(1+\frac{1}{T_{i} s}+T_{d} s\right)
$$

A ação de controle aplicada ao sistema é a combinação das três ações de controle individuais, e o ajuste dos ganhos de cada ação de controle é chamada de sintonia. Sendo assim, o ganho proporcional $\left(K_{p}\right)$, visa diminuir o tempo de acomodação enquanto que o termo integrativo $\left(T_{i}\right)$, é o tempo para que a saída do integrador atinja o valor de ajuste. Completando o sistema, o termo derivativo $\left(T_{d}\right)$ é o intervalo de tempo que a ação de controle derivativa antecede a ação de controle proporcional.

A Figura 6 representa o diagrama em blocos do controlador PID proposto neste trabalho. O erro provém da diferença entre a posição lida do pêndulo $(\theta)$ e a referência $\left(\theta^{\prime}\right)$ admitida ao inicializar o processo. Tal variável é aplicada como entrada do controlador.

A saída do bloco PID é então apresentada ao modelo do sistema, e este por sua vez determina um novo valor de ângulo.

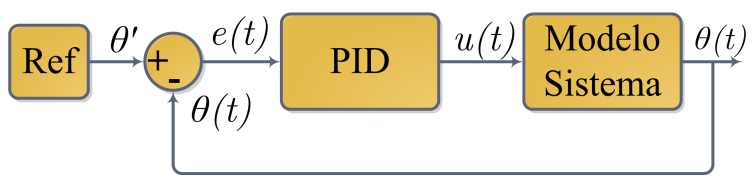

Figura 6: Diagrama do controlador PID

A implementação do controlador no bloco programável é realizado no processamento digital $A R M 7^{\mathrm{TM}}$, e para isso faz-se necessário a discretização da malha de controle.

Assim utiliza-se como base o método de aproximação backward para o termo derivativo e o trapezoidal para o termo integrativo, conforme as equações (11) e (12) onde $T_{s}$ representa o período de amostragem (Franklin et al., 1998).

$$
\begin{gathered}
s=\frac{(z-1)}{T_{s} z} \\
s=\frac{2}{T_{s}} \frac{(z-1)}{(z+1)}
\end{gathered}
$$

Substituindo a equação (11) na variável $(s)$ do termo integrativo da equação (10), e a equação (12) no termo derivativo, reescreve-se os termo integrador e derivativo discretos conforme as equações (13) e (14), respectivamente. 


$$
\begin{gathered}
\frac{1}{T_{i} s}=\frac{1}{T_{i}\left(\frac{2}{T_{s}} \frac{(z-1)}{(z+1)}\right)} \\
T_{d} s=T_{d} \frac{(z-1)}{T_{s} z}
\end{gathered}
$$

Agrupando os termos da equação (13) e (14) e realizando as devidas manipulações matemáticas, obtém-se a equação das diferenças do controlador PID, mostrado na equação (15), onde $a=K_{p}$, $b=K_{p}\left(T_{s} / T_{i}\right)$ e $c=K_{p}\left(T_{d} / T_{s}\right)$.

$$
\begin{aligned}
& u[n]=u[n-1]+a(e[n]-e[n-1])+ \\
& \frac{b}{2}(e[n]+e[n-1])+ \\
& \quad c(e[n]-2 e[n-1]+e[n-2])
\end{aligned}
$$

Considerando o modelo proposto na Figura 6, é possível realizar a sintonia pelo método do lugar das raízes, onde são encontradas os valores dos respectivos ganhos. Para implementação, após um ajuste fino, estes valores são apresentados na Tabela 3 .

Tabela 3: Parâmetros de controle

\begin{tabular}{cc}
\hline \hline Constante & Valor \\
\hline$K_{p}$ & 19 \\
$T_{i}$ & 2 \\
$T_{d}$ & 0,0035 \\
$T_{s}$ & $3 \mathrm{~ms}$ \\
\hline \hline
\end{tabular}

Utilizando a equação (15) e os termos da Tabela 3, cria-se a rotina do controlador PID para estabilização da haste do pêndulo.

\subsection{Controlador Fuzzy}

A lógica nebulosa é muitas vezes descrita como uma ampliação da lógica clássica, oferecendo uma alternativa para a representação do conhecimento, levando em consideração as imprecisões e incertezas contidas no sistema.

Sendo assim, uma característica relevante é a presença de um especialista, que conhece os detalhes e o método para realizar o controle adequado do sistema.

A representação do conhecimento é um ramo da Inteligência Artificial (IA) que visa transcrever os conceitos deste especialista em uma rotina de controle automático, mantendo as especificidades de cada sistema (Zadeh, 1983).

O projeto do controlador fuzzy em uma primeiro estágio é baseado nos conhecimentos do especialista e após alguns testes, suas regras de controle são ajustadas até encontrar o ponto ideal.

O projeto de uma controlador fuzzy segue basicamente a seguinte sequência (Lee, 1990):
- Determinar as entradas e saídas do controlador;

- Definir o universo de discurso para cada variável utilizada;

- Estipular as regras de controle;

- Analisar o melhor método de defuzzificação;

- Realizar teste do controlador e pequenos ajustes.

Sendo assim, define-se o erro de posição do pêndulo em relação a base e a derivada do erro como entradas para o controlador nebuloso.

Pode-se dizer que uma variação de ângulo da haste de \pm 5 graus em relacão a referência possui uma dinâmica linear. Desta forma, considera-se que a variação do erro de posição do pêndulo está entre $(-\pi / 36)$ a $(\pi / 36)$, conforme apresentado na Figura 7.

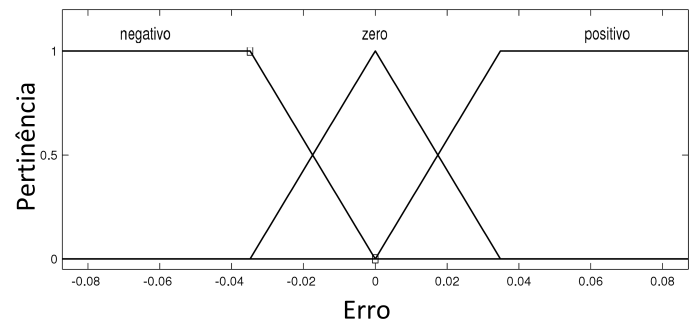

Figura 7: Função de pertinência erro

A variação da derivada do erro ocorre entre $(-\pi / 90)$ a $(\pi / 90)$, evitando assim que o pêndulo desloque rapidamente, conforme descrito na $\mathrm{Fi}$ gura 8.

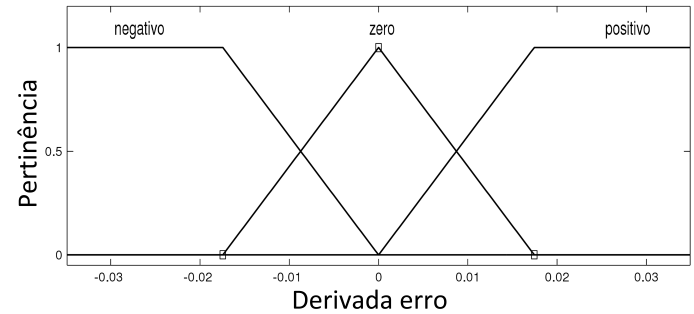

Figura 8: Função de pertinência da derivada do erro

Já para a saída do controlador, define-se a força horizontal aplicada a base móvel do pêndulo, que possui um universo de discurso entre -100 e 100, como representado na Figura 9.

Após a definição das entradas e saída do controlador, faz-se necessário a criação das regras de implicação, que relacionam as funções de pertinência das entradas com as saídas, conforme as regras apontadas pelo especialista.

Para implementação do controlador fuzzy foram criadas nove regras de implicação, as quais são descritas na Tabela 4. 


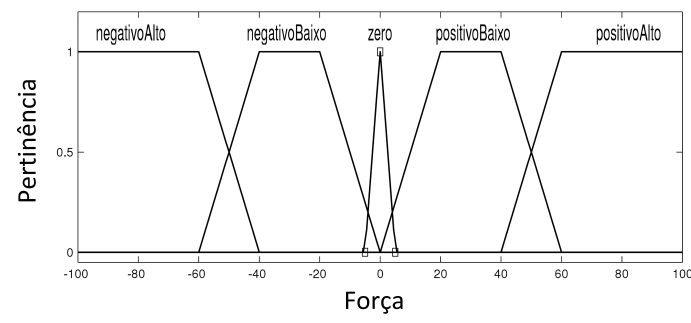

Figura 9: Função de pertinência da força

Tabela 4: Regras do Controlador Fuzzy

\begin{tabular}{|c|c|}
\hline Regra & Descrição \\
\hline 1 & $\begin{array}{l}\text { If erroÂngulo is negativo and devErro is } \\
\text { negativo then força is negativoAlto. }\end{array}$ \\
\hline 2 & $\begin{array}{l}\text { If erroÂngulo is negativo and devErro is } \\
\text { zero then força is negativoBaixo. }\end{array}$ \\
\hline 3 & $\begin{array}{l}\text { If erroÂngulo is negativo and devErro is } \\
\text { positivo then força is zero. }\end{array}$ \\
\hline 4 & $\begin{array}{l}\text { If erroÂngulo is zero and devErro is } \\
\text { negativo then força is negativoBaixo. }\end{array}$ \\
\hline 5 & $\begin{array}{l}\text { If erroÂngulo is zero and devErro is } \\
\text { zero then força is zero. }\end{array}$ \\
\hline 6 & $\begin{array}{l}\text { If erroÂngulo is zero and devErro is } \\
\text { positivo then força is positivoBaixo. }\end{array}$ \\
\hline 7 & $\begin{array}{l}\text { If erroÂngulo is positivo and devErro is } \\
\text { negativo then força is zero. }\end{array}$ \\
\hline 8 & $\begin{array}{l}\text { If erroÂngulo is positivo and devErro is } \\
\text { zero then força is positivoBaixo. }\end{array}$ \\
\hline 9 & $\begin{array}{l}\text { If erroÂngulo is positivo and devErro is } \\
\text { positivo then força is positivoAlto. }\end{array}$ \\
\hline
\end{tabular}

O operador de implicação utilizando no controle foi o MIN, onde o mínimo valor de pertinência das entradas são repassados para a saída. Já para a defuzzificação, foi utilizado o método conhecido como Centro das Áreas (CA), que encontra o centro de massa do conjunto de saída calculado pelo valor mínimo das pertinências de entrada.

Assim como descrito na Secção 4.1, o controlador fuzzy também é aplicado ao sistema em malha fechada. Substituindo, para tal, o bloco do controlador PID pelo conjunto do controlador fuzzy, como pode ser observado na Figura 10.

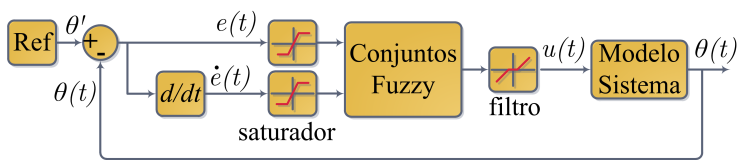

Figura 10: Diagrama do controlador nebuloso

A diferença deste modelo para o modelo PID é a utilização de mais uma variável de entrada. Desta forma aplica-se a derivada do erro $(\dot{e}(t))$ juntamente com o erro $(e(t))$ para construção do sinal do atuador. Sendo que a variação do sinal de entrada do conjunto fuzzy não deve superar o universo de discurso estabelecido, este sinal é limitado pelo bloco saturador de cada entrada.

Considerando que o controlador nebuloso pode apresentar saídas com valores pequenos e não representativos, uma solução proposta, foi a utilização de uma função rejeita faixa. Este filtro elimina o acúmulo do sinal do atuador, rejeitando valores com o módulo inferiores a $10^{-5}$ e considerando a saída do controlador como zero.

\section{Metodologia}

Para determinar o melhor método de controle, algumas análises foram desenvolvidas considerando a variação do ângulo da haste e o sinal do controlador. Após a implementação física do pêndulo invertido sobre carro com a validação dos métodos de controle, o modelo matemático do sistema é utilizado para geração dos dados de resposta e sinais dos atuadores.

\section{1 Índices de desempenho}

Para mensurar a eficiência do controlador, foram adotados alguns índices de desempenho. O índice $S u$ representa a variação média quadrática da variável manipulada pelo controlador, conforme descrito na equação (16).

$$
S u=\frac{1}{n+1} \sum_{k=1}^{n} \Delta u^{2}(k)
$$

O índice ITSE é a integral do erro quadrático em relação ao tempo decorrido de controle, conforme a equação (17).

$$
\text { ITSE }=\sum_{k=1}^{n} k T e(k)^{2}
$$

O índice $R B M S E M C E$, por sua vez, é definido como o erro médio quadrático mais o esforço realizado pelo controlador, sendo descrito pela equação (18).

$$
R B M S E M C E=\frac{1}{n} \sum_{k=1}^{n} e(k)^{2}+\frac{\beta}{n} \sum_{k=1}^{n} u(k)
$$

Desta forma, três métricas de comparação são utilizadas para avaliar o desempenho dos métodos de controle. Cada qual, tendo um parâmetro de cálculo em específico.

\subsection{Análise de resultados}

Para o cálculo dos índices da Tabela 5, foi aplicado ao sistema com o controlador PID e fuzzy o mesmo distúrbio, e os índices foram calculados sobre as resposta de cada sistema.

Sendo assim, o sinal do sistema quando utilizado o controlador PID e fuzzy é mostrado na Figura 11. 


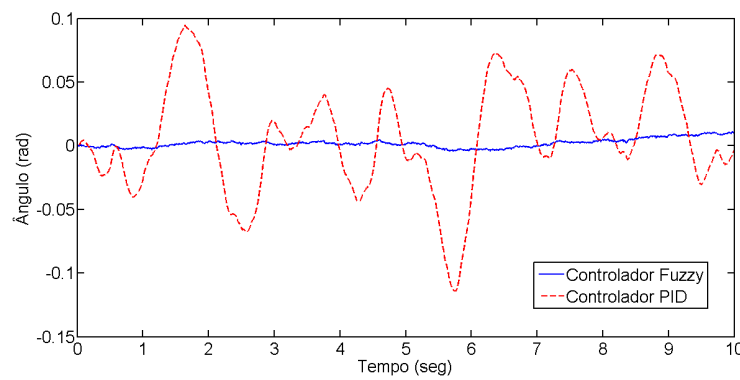

Figura 11: Resposta ao distúrbio utilizando controlador PID e fuzzy

Analisando os resultados apresentados na Tabela 5, observa-se que a resposta do sistema utilizando o controlador fuzzy apresenta uma variação média $(S u)$ maior em relação ao controlador PID. Tal característica se justifica por manter-se o deslocamento do pêndulo com o mínimo valor possível, resultando em respostas mais rápidas e oscilantes.

Tabela 5: Índice de desempenho dos controladores

\begin{tabular}{lcc}
\hline \hline Indice & $P I D$ & Fuzzy \\
\hline Su & 7,96 & $9,9.10^{4}$ \\
ITSE & 29,43 & 0,415 \\
RBMSEMCE & 0,91 & 0,62 \\
\hline \hline
\end{tabular}

Já nos índices (ITSE) e ( $R B M S E M C E)$ que utilizam a integral do erro como base, o controlador fuzzy deve ser destacado, pois produz valores menores quando comparados com o controlador PID.

\section{Conclusão}

Neste trabalho foi apresentado a modelagem e o controle, pelo método convencional e nebuloso, de uma sistema dinâmico com características nãolineares. O controle nebuloso é definido de forma otimizada, utilizando nove regras de implicação.

A comparação da resposta do sistema quando aplicado o controlador PID e fuzzy é realizada, onde o controlador nebuloso mostrou-se mais eficiente para o controle do ângulo do pêndulo invertido sobre carro. Tal estratégia desmostrou os menores valores nos índices que medem o erro quadrático médio.

As duas metodologias de controle propostas são validadas com a implemetadas no bloco programável do kit Lego Mindstorms $\mathrm{NXT}^{\circledR}$, considerando-se o tempo de processamento satisfatório. Cabe ressaltar que o tempo de execução de cada rotina é considerado no modelamento matemático.

\section{Agradecimentos}

Este trabalho é financiado pela FINEP - Financiadora de Estudos e Projetos (Processo No 4971/2006) e CNPq (Processo No 473611/20112,06/56093). O mesmo tem o apoio da Fundação de Apoio à Educação, Pesquisa e Desenvolvimento Científico e Tecnológico da UTFPR (FUNTEF), da Fundação Araucária de Apoio ao Desenvolvimento Científico e Tecnológico do Paraná, Conselho Nacional de Desenvolvimento Científico e Tecnológico - CNPq Brasil e bolsas Capes-DS.

\section{Referências}

Dorf, R. e Bishop, R. (1998). Modern control systems, $8^{\text {a }}$ edição, Addison-Wesley, Indianapolis.

Faizan, F., Farid, F., Rehan, M., Mughal, S. e Qadri, M. (2010). Implementation of discrete pid on inverted pendulum, Education Technology and Computer (ICETC), 2010 2nd International Conference on, Vol. 1, pp. 48-51.

Franklin, G., Powell, J. e Workman, M. (1998). Digital control of dynamic systems, AddisonWesley world student series, Addison-Wesley.

Fu, Y.-C. e Lin, J.-S. (2005). Nonlinear backstepping control design of the furuta pendulum, pp. $96-101$.

Furuta, K., Nishihara, H. e Mori, S. (1976). Control of unstable mechanical system control of pendulum, International Journal of Control 23: 673-692. DOI: 10.1080/00207177608922192

Gonçalves, J., Lima, J., Malheiros, P. e Costa, P. (2010). Sensor and actuator stochastic modeling of the lego mindstorms nxt educational kit, In 10th Conference on Mobile Robots and Competitions. Leiria, pp. 11-16.

Krishnan, R. (2001). Electric motor drives: modeling, analysis, and control, Pennsylvania State University : Prentice Hall.

Lee, C. (1990). Fuzzy logic in control systems: fuzzy logic controller - Part I, Systems, Man and Cybernetics, IEEE Transactions on 20(2): $404-418$.

Morais, M. H. E., Muralikrishna, A., Bravo, R. e Guimarães, L. N. F. (2005). Um controlador nebuloso aplicado ao problema do pêndulo invertido, XXVIII Congresso Nacional de Matemática Aplicada e Computacional, São Paulo, Vol. 1, pp. 1-5.

Nise, N. S. (2002). Engenharia de Sistema de Controle, $3^{\text {a }}$ edição, Editora LTC, Rio de Janeiro. 
Nour, M., Ooi, J. e Chan, K. (2007). Fuzzy logic control vs. conventional pid control of an inverted pendulum robot, Intelligent and $\mathrm{Ad}$ vanced Systems, 200\%. ICIAS 200\%. International Conference on, pp. 209-214.

Ogata, K. (2010). Engenharia de Controle Moderno, $5^{\mathrm{a}}$ edição, Pearson Prentice Hall, São Paulo.

Wu, J., Su, H. e Wu, T. (2008). Ann control of inverted pendulum, Intelligent Networks and Intelligent Systems, 2008. ICINIS '08. First International Conference on, pp. 9-12.

Yadav, A., Gaur, P., Mittal, A. e Anzar, M. (2011). Comparative analysis of various control techniques for inverted pendulum, Power Electronics (IICPE), 2010 India International Conference on, pp. 1-6.

Zadeh, L. A. (1983). Commonsense knowledge representation based on fuzzy logic, Computer 16(10): 61-65. DOI: 10.1109/MC.1983.1654199 